\title{
Zero Moment Point Based Pace Reference Generation for Quadruped Robots via Preview Control
}

\author{
Tunc Akbas, S. Emre Eskimez, Selim Ozel, O. Kemal Adak, Kaan C. Fidan and Kemalettin Erbatur \\ The authors are with the Faculty of Engineering and Natural Sciences, Sabanci University, Istanbul, 34956 Turkey (phone: +90- \\ 216-483-9585; fax: +90-216-483-9550; e-mail: tuncakbas@sabanciuniv.edu, eskimez@sabanciuniv.edu, selimozel \\ @sabanciuniv.edu, kaancfidan@sabanciuniv.edu, erbatur@sabanciuniv.edu).
}

\begin{abstract}
Legged robots have significant advantages over other types of mobile robots when task at hand requires the robot to overcome obstacles. This paper presents a reference trajectory generation method for a quadruped robot for pace gait on a flat surface. The approach is based on the Zero Moment Point (ZMP) stability criterion and the Linear Inverted Pendulum Model (LIPM). ZMP reference trajectories for pace is proposed, from which reference trajectories for the Robot Center of Mass (CoM) references are obtained by applying preview control. The position of leg joints are computed using inverse kinematics according to CoM reference trajectory. Proposed reference trajectory generation synthesis is tested through full-dynamics 3D simulation. A 16-degrees-of-freedom (DOF) quadruped robot model is used in the simulations. Simulation results show the success of the reference generation technique for the pace gait.
\end{abstract}

\section{INTRODUCTION}

Quadruped robots have advantages in search and rescue operations, in hazardous environments and in load carrying tasks. The quadruped structure is versatile, combining advantages of speed and climbing abilities. Compared to wheeled and tracked mobile robots, they are better in adapting to rough and steep terrains. They can be employed for applications which take place on surface profiles ranging from flat ground to hard to cross terrain. Reference generation in this field is one of the central design steps.

There are various gait types for walking of quadruped robots [1]. Gait types differ from each other by swing order of the legs, support and swing periods and timing. There are various gaits that are observed in locomotion of four legged animals. Quite common ones are crawl, trot, pace, canter and gallop. Crawl is generally observed in turtles. It uses three contact points during the swing period, enhancing stability. Leg swings are in the left front - right rear - right front - left rear order. Trot gait can be observed mostly in camels and horses, also in many other four legged animals. In trot, front and rear legs at different sides are swung at once [1]. Trot has been the subject of many quadruped robot researches [2-7]. Pace is a gait which is similar to trot. In pace, legs at the same side are swung together. This gait can be observed in lizards. Canter and gallop are faster gaits which are generally observed in horses.

There are various methods to obtain gait patterns for quadruped robots. Central pattern generation is one of these methods [8, 9]. Another method is autonomous evolution of gait patterns [10]. Finding gait patterns by hybrid optimal control is discussed in [11]. ZMP based energy efficient gait pattern generation methods are proposed too $[5,12]$.

ZMP based reference trajectory generation is widely employed for biped robots [13-20]. There are studies reported on ZMP based locomotion references for quadruped robots too [3-7,21-25]. The ZMP stability criterion states that, in order to maintain the stability of the robot, the ZMP should be kept in supporting polygon defined by foot or feet touching the ground, ZMP equations can be obtained easily for robots with many DOFs, but the amount of position, velocity and acceleration variables that are included in these equations make it difficult to employ them in the synthesis of a reference trajectory. This is why many researches use more simplified models instead of ones with many DOFs. LIPM is such a simplified model. The first step in reference synthesis is the assignment of foot landing positions for a desired gait. A ZMP reference which is consistent with the foot landing positions is generated next. Afterwards a reference trajectory for the robot CoM is generated using the ZMP reference trajectory and the LIPM. Robot joint position references are computed by solving an inverse kinematics problem between feet landing positions and robot CoM. These position references are tracked using independent PID joint controllers. In addition to PID joint controllers, online reference modifications with feedback from inertial sensors and ankle mounted force/torque sensors are employed for control approach in most of the studies.

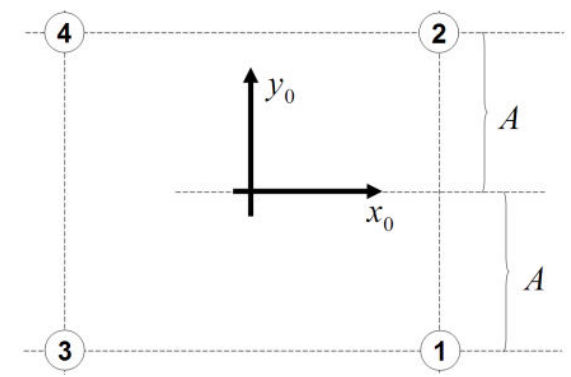

Figure 1. Foot positions of the quadruped before starting the locomotion. Circles represent feet and numbered accordingly: (1) front right foot, (2) front left foot, (3) rear right foot and (4) rear left foot. $x_{0}$ are $y_{0}$ variables that define the world coordinate frame. 
(1)

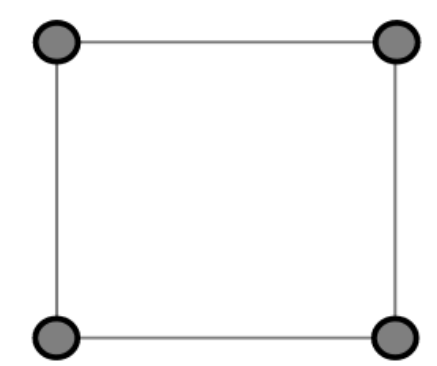

(3)

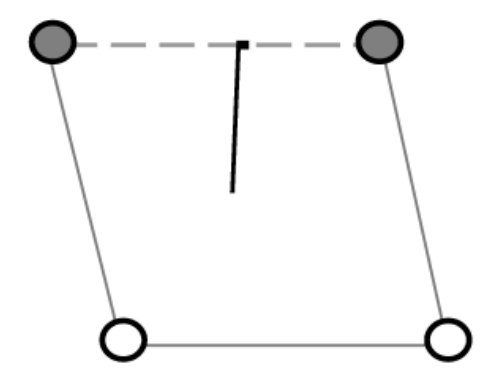

(5)

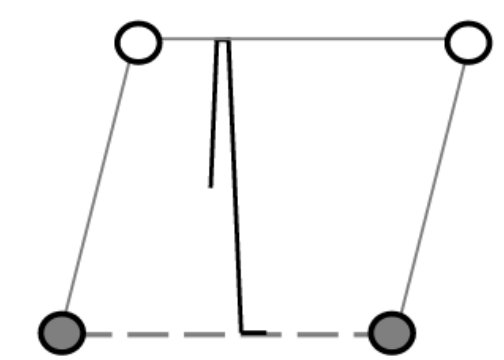

(7)

(9)

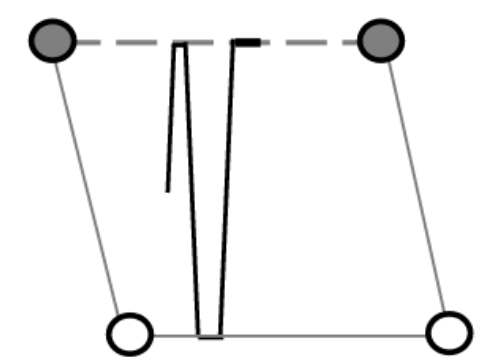

(11)
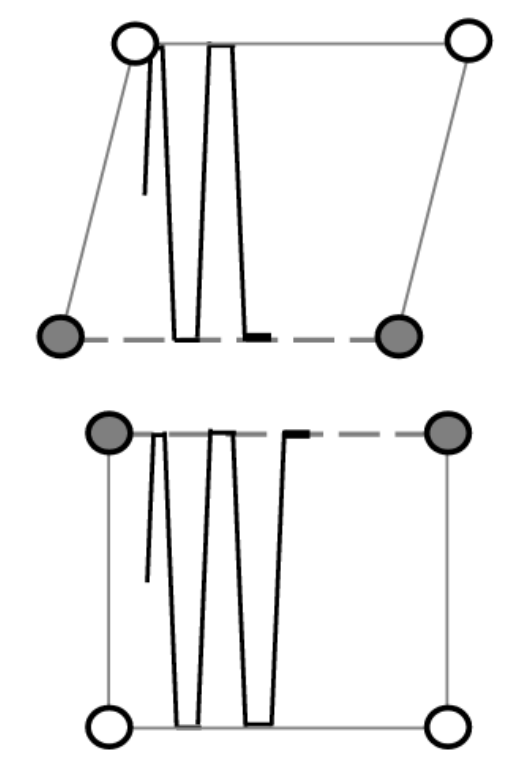

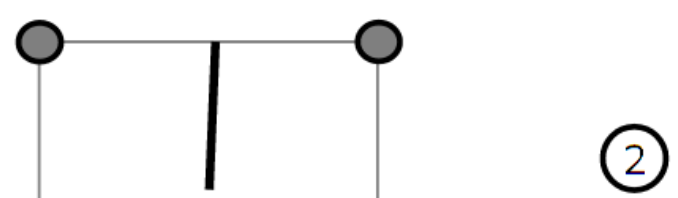

(4)

(6)

(8)
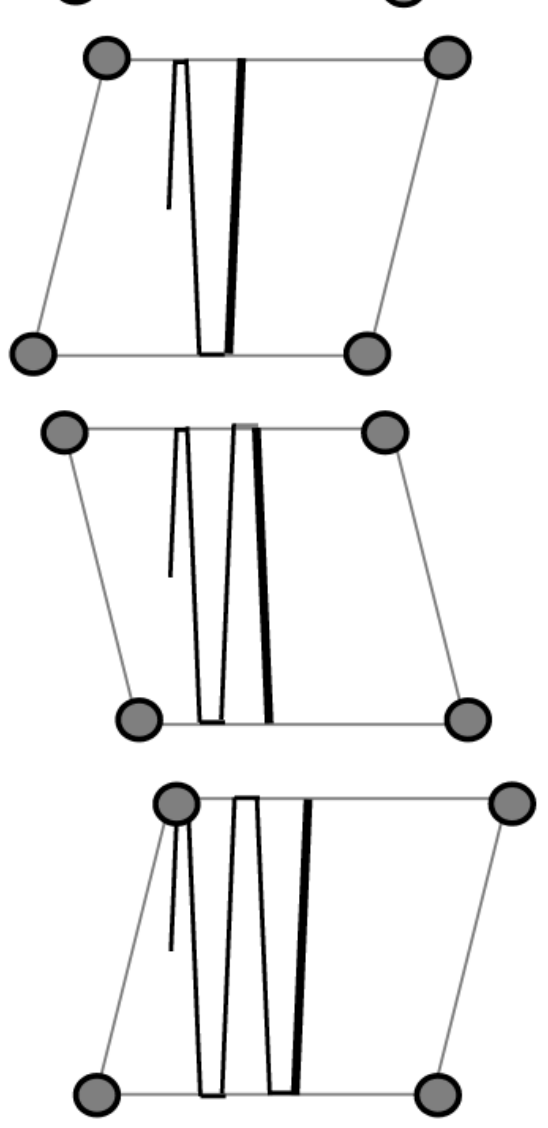

(10)

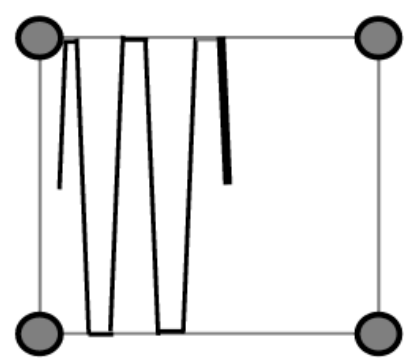

Figure 2. Construction of ZMP references for five step pace gait. The numbers in the circles indicate the phases of the locomotion. The dark circles represent the supporting feet. The thin solid line shows the ZMP reference curve. The thick solid line indicates the ZMP reference at a given support period. Dashed line shows the line between supporting feet in the two feet support phase. The rectangular and diamond shapes mark the support polygon in quadra support phases. 
There are various methods for obtaining CoM references from ZMP references. Laplace transformation [15, 17], Fourier Series approach $[13,18,26]$ and preview control $[17,27]$ are among methods. Preview control of ZMP in biped robots is introduced by [17]. Preview control based reference generation is a well accepted method since it allows online manipulation of ZMP references. However, preview control is not commonly applied for reference trajectory synthesis of robots which have more than two legs. One of the few examples is Asterisk, a hexapod robot, which uses preview control method to create its CoM reference trajectory, while rolling on the ground [24].

In [25], the ZMP criterion and preview control are used during cross leg support phases of a quadruped robot, but gait generation process is not defined explicitly. This paper differs from [25] by proposing a novel method which makes a connection between preview control of ZMP trajectory and a pace gait generation technique. It can be applied to other gaits as well [28].

An earlier work of the authors of this paper applies the ZMP stability criterion and preview control technique with the LIPM for the quadruped robot trot reference generation [28]. This paper extends the results in [28] further by generating a gait reference for pace. Therefore the preview control technique in [28] is generalized for different gait types in this paper. Proposed reference synthesis and control structure are applied on a 16-DOF quadruped robot model using a full-dynamics 3D simulation.

The paper is structured as follows: The computation of ZMP reference trajectory for pace gait is discussed in Section II. A summary of LIPM and computation of robot CoM reference trajectory can be found in Section III. Simulation results are presented in Section IV. Section V concludes the paper.

\section{ZMP REFERENCE TRAJECTORY FOR PACE}

Throughout the paper $x$-direction of a world coordinate frame is the walking direction of the quadruped robot model, $y$ axis is perpendicular to walking direction and $z$-axis is perpendicular to ground surface. There is another coordinate frame attached to the body of the quadruped robot which has the same axis alignment as world coordinate frame except that there is a $z$-axis offset. $y$-axis and $x$-axis of the world coordinate frame are shown in Fig. 1.

During pace, leg pairs of the quadruped swing together. These pairs are 1-3 and 2-4 with respect to the notation given in Fig. 1. While a pair of legs is swinging, a support line segment emerges between remaining two legs. ZMP reference trajectory should be generated such that it should not get out of this line segment in order to achieve stable locomotion. It should be noted that in the literature the ZMP criterion is generally considered with a support polygon, rather than with a support line segment. However, it is very typical for real applications that a narrow strip between the two supporting feet, rather than a perfect line segment, supports the robot body.

Foot placement locations and timings must be determined in order to obtain a ZMP reference trajectory. Construction of foot placement locations for five steps of the ZMP reference trajectory is shown in Fig. 2. It is to be noted that the pace necessitates non-zero duration full-support. This is because the support line segments (emerges between legs that are in contact with the ground) in the two feet support phases do not cross each other. The ZMP should travel between the support line segments, and quadra support phase has to be allocated for this travel, so that ZMP trajectory is continuous.

ZMP reference trajectories in Fig. 2 are described in $x$-y plane. However time domain references of $x$ and $y$ directions of ZMP trajectories are needed for the computation of CoM trajectories. In order to get time domain references, constant ZMP reference speed is assumed in the walking direction $(x-$ direction). Speed is derived from the desired locomotion parameters: Step length, double support phase duration and quadra support phase duration. The distance covered by the ZMP in the quadra support and double support phases are proportional to the duration of these phases.

In Fig. 1 during Phase 1 the quadruped is at a standing position on a rectangular support polygon and ZMP is at the center of this support polygon. At the beginning of Phase 2 the ZMP trajectory moves to the line passing through feet 4 and 2 (feet numberings are defined in Fig. 1). The movement of ZMP trajectory ends when it reaches this line. This positioning is a preparation for the next swing phase, namely, Phase 3. The ZMP trajectory also moves forward ( $x$-direction) in Phase 2 since It is required for generating suitable time domain references for $\mathrm{CoM}$ trajectory computation as mentioned above. During Phase 3 the robot is supported by feet 2 and 4. The ZMP moves forward in the $x$-direction along the line joining feet 4 and 2. Phase 3 ends when swing legs come in contact with the ground. At Phase $4 \mathrm{ZMP}$ starts to move towards the line passing through feet 3 and 1 . Phase 4 ends when ZMP reaches this line. After Phase 4 the rest of the ZMP trajectory of quadruped becomes periodic until Phase 11(last double support phase). Even numbered labels in Fig. 2 show quadra support phases. Likewise, odd numbered labels show double support phases. At Phase 11 swinging legs cover a distance of half the step length in order to become aligned with the supporting legs in the $x$-direction. At the last phase ZMP trajectory moves towards the middle of the support polygon and locomotion ends.

\section{Computation of Robot CoM Using PREview CONTROL}

The LIPM is a considerably simple model which can be easily employed for the reference generation of legged robots. Trunk of the robot is approximated as a point mass located at the CoM of the robot. This point is connected to a non-slipping point on the ground by a massless rod. The massless rod is an ideal model for the supporting leg. Swinging legs are also assumed to be massless. Hence the point mass and the robot CoM coincide. The LIPM is illustrated in Fig. 3. In the figure $c=\left(\begin{array}{lll}c_{x} & c_{y} & c_{z}\end{array}\right)^{T}$ are the coordinates of the point mass. $c_{z}$ is assumed to be constant. With this assumption, the relation 
between the robot ZMP and CoM on $\mathrm{x}$ and $\mathrm{y}$ directions become decoupled [17]:

$$
\begin{aligned}
& p_{x}=c_{x}-\left(z_{c} / g\right) \ddot{c}_{x} \\
& p_{y}=c_{y}-\left(z_{c} / g\right) \ddot{c}_{y}
\end{aligned}
$$

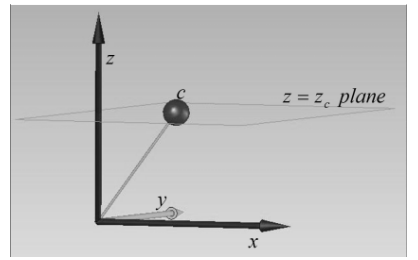

Figure 3. Linear Inverted Pendulum Model

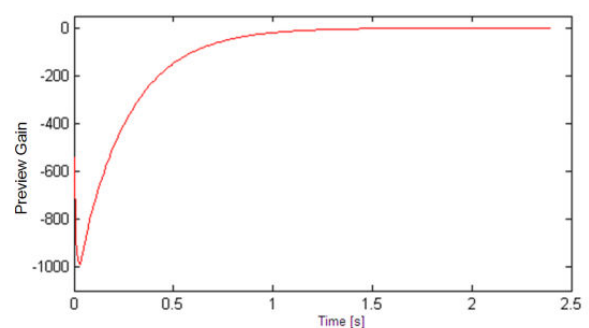

Figure 4. Preview control gain $G_{p}(j)$ for 2.5 seconds ( $T=0.01 \mathrm{~s}$,

$$
z_{c}=0.614 \mathrm{~m}, Q_{e}=1, Q_{x}=0, R=10^{-6} \text { ) }
$$
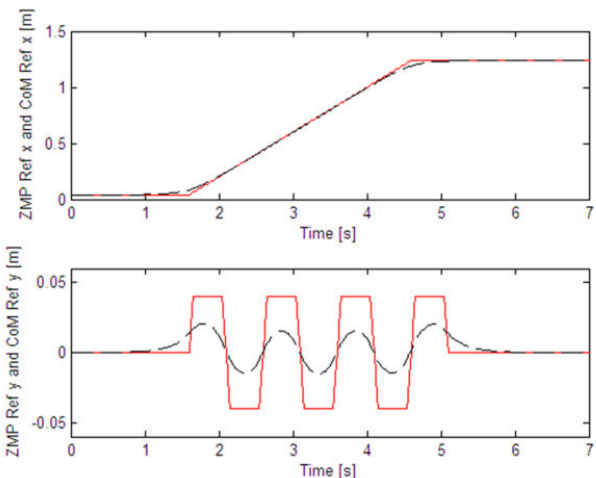

Figure 5. Corresponding ZMP and CoM reference trajectories for seven steps pace.

In equations (1) and (2), $g$ is the gravitational constant, $z_{c}$ is the height of the plane on which the motion of the point mass is constrained. $p_{x}$ and $p_{y}$ are ZMP coordinates.

Computing the robot CoM from a ZMP reference trajectory is an important problem. [17] proposes a solution to this problem using preview control. The solution uses the following system description.

$$
\begin{gathered}
\frac{d}{d t}\left[\begin{array}{l}
c_{x} \\
\dot{c}_{x} \\
\ddot{c}_{x}
\end{array}\right]=\left[\begin{array}{lll}
0 & 1 & 0 \\
0 & 0 & 1 \\
0 & 0 & 0
\end{array}\right]\left[\begin{array}{l}
c_{x} \\
\dot{c}_{x} \\
\ddot{c}_{x}
\end{array}\right]+\left[\begin{array}{l}
0 \\
0 \\
1
\end{array}\right] u_{x} \\
p_{x}=\left[\begin{array}{lll}
1 & 0 & -z_{c} / g
\end{array}\right]\left[\begin{array}{lll}
c_{x} & \dot{c}_{x} & \ddot{c}_{x}
\end{array}\right]^{T}
\end{gathered}
$$

The control input $u_{x}$ in the system is the first derivative of robot CoM acceleration with respect to time. Output of the system is the ZMP reference position on $x$ coordinate. Previously computed (Section II) ZMP reference trajectories are tracked by control input. As a result the system computes the robot CoM reference trajectory as state variables, which forces the system to track the ZMP reference trajectory. Equations (3) and (4) need to be converted to discrete time in order to apply preview control to the system:

$$
\begin{gathered}
x_{k+1}=A x_{k}+B u_{k} \quad p_{k}=C x_{k} \\
x_{k}=\left[\begin{array}{lll}
c_{x}(k T) & \dot{c}_{x}(k T) & \ddot{c}_{x}(k T)
\end{array}\right]^{T} \quad u_{k}=u_{x}(k T) \\
p_{k}=p_{x}(k T) \\
A=\left[\begin{array}{ccc}
1 & T & T^{2} / 2 \\
0 & 1 & T \\
0 & 0 & 1
\end{array}\right] \\
B=\left[\begin{array}{lll}
T^{3} / 6 & T^{2} / 2 & T
\end{array}\right]^{T} \quad C=\left[\begin{array}{lll}
1 & 0 & -z_{c} / g
\end{array}\right]
\end{gathered}
$$

$k$ is a discrete time index. $T$ is the sampling period. The system description in equations (5) and (7) is employed for the $y$ directional motion too. For this, the state variable and the system output are replaced by $x_{k}=\left[\begin{array}{lll}c_{y}(k T) & \dot{c}_{y}(k T) & \ddot{c}_{y}(k T)\end{array}\right]^{T} \quad$ and $\quad p_{k}=p_{y}(k T)$, respectively. Calculations for $x$ and $y$ coordinates are done separately. Preview control differs from traditional control methods such that it looks forward in time at reference values for $N_{L}$ cycles and calculates an optimal control input which minimizes a performance index for that time period. Rosenthal and Tomizuka developed a method in order to calculate the optimal control input for discrete time systems [29]. This method is completed by Katayama [30]. Optimization of $u_{k}$ function which controls the system is computed with the following performance index:

$$
J=\sum_{i=k}^{\infty}\left\{Q_{e} e(i)^{2}+\Delta x^{T}(i) Q_{x} \Delta x(i)+R \Delta u^{2}(i)\right\}
$$

The calculation of control input and details of the performance index (8) are explained in detail [30]. In (8), $e(i)=p_{i}-p_{i}^{r e f}$ is the tracking error between system Output 
and the reference. $\Delta x_{k}=x_{k}-x_{k-1}$ is the vector showing the changes of the state variables and $\Delta u_{k}=u_{k}-u_{k-1}$ is the vector that shows changes in input.

$Q_{e}$ and $R$ are scalar variables greater than zero ( in multiple input/output systems these variables will be matrices) and $Q_{x}$ is $3 \times 3$ a symmetric non-negative definite matrix. While considering a preview control that looks $N_{L}$ cycles forward in every sampling time, the optimal input which minimizes the performance index in (8) is given by $[17,30]$

$$
u_{k}=-G_{i} \sum_{i=0}^{k} e(k)-G_{x} x(k)-\sum_{j=1}^{N_{L}} G_{p}(j) p^{r e f}(k+j) .
$$

First term in (9) is the integral action over the tracking error and second term is state feedback. Preview control term, namely third term, deserves further explanation. It uses the reference values which are $j$ cycles in front of the current control cycle and integrates these values with a time varying gain function and adds the result to the overall controller. It can be observed from Fig.4 that $j$ values closer to current cycle have more importance. This observation implies that an event in future cycles is taken into account when it is close enough to the present cycle.

In (9), $G_{i}, G_{x}$ and $G_{p}(j)$ are the gains calculated from the weights $Q_{e}, Q_{x}, R$ and the system parameters of equation (5). As seen in Fig. 4 preview control gain $G_{p}(j)$ converges to zero after 1.6 seconds. Therefore selecting $N_{L}$ as $1.6 / T$ is a good choice as mentioned in [17]. During this process robot CoM trajectory is obtained by saving values of system state vector.

The ZMP reference trajectories for the pace gait and corresponding robot $\mathrm{CoM}$ reference calculated by preview control are shown in Fig. 5.

\section{Simulation Results}

Quadruped robot model employed in this paper has 4 DoFs at each leg. There are two DoF at the hip. Knee and ankle are single-DoF joints. The kinematic arrangement is shown in Fig. 7. A view of animation environment can be seen in this figure too. Link lengths and joint dimensions are shown in Table I. Simulations are run using reference trajectories, obtained by methods explained in Sections II- III. Joint position references are obtained by solving inverse kinematics problem between $\mathrm{CoM}$ and Cartesian foot position references. These position references are tracked using independent PID joint controllers. A block diagram of the controllers used in the simulation is given in Fig 6. Adaptive penalty based system is used to simulate ground contacts of the simulation. [31] explains the details of simulation algorithm and ground contact model.

The foot trajectories as expressed in the world coordinate frame complete the locomotion references. Mainly, sinusoidal functions are used for the foot $x$ and $z$ directional references for smooth motion. The $y$-directional foot references are constants (at $A$ for left hand).
Variables for reference generation are shown in Table II. As an example of simulation results, Fig. 8 shows a portion of walking period which indicates the CoM reference trajectory and actual CoM trajectory of the quadruped with respect to world coordinate frame. Since LIPM model is a simplified model, exact reference tracking is not expected. The difference between CoM reference trajectory and actual CoM trajectory is tolerable for a steady walk. Also, Fig 9 shows roll and pitch angles of the trunk of the robot during a pace gait. Amplitude of these angles demonstrates a steady and successful walk.

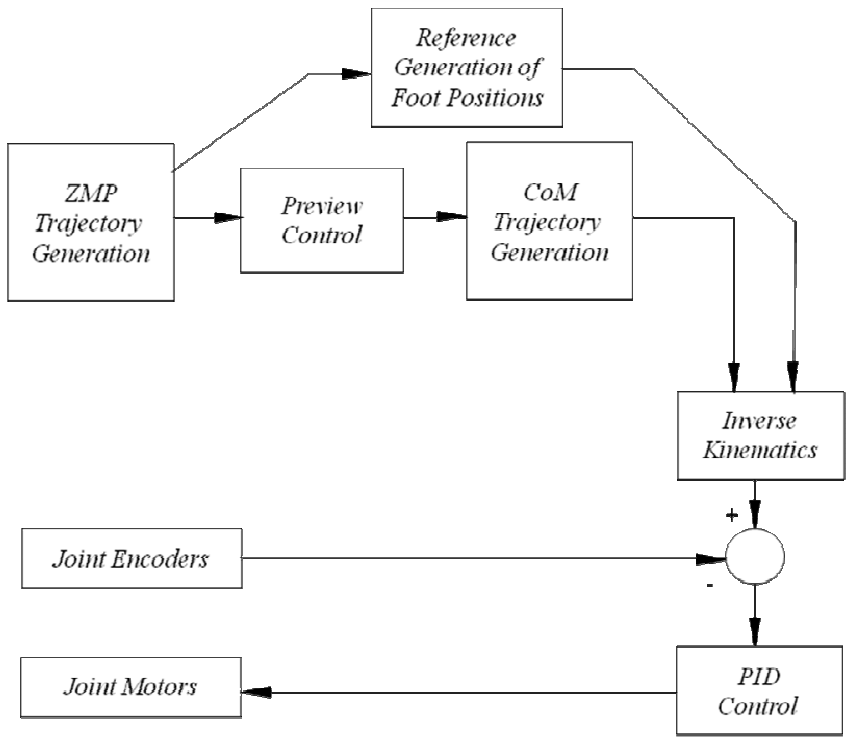

Figure 6. The control block diagram.

\section{CONCLUSION}

Stable ZMP reference trajectories for pace gait of a quadruped robot are generated in this paper. The relationship between ZMP and robot CoM is modeled by LIPM. Preview control is used to create robot CoM reference trajectories. Joint references are obtained by solving inverse kinematics problem between robot CoM and feet position references. Locomotion simulation studies of a quadruped robot are run with the proposed ZMP based reference trajectory generation technique. Simulated robot has $16 \mathrm{~cm}$ step lengths and has walked successfully with pace and trot gaits. Simulation results motivate the application of the presented reference generation approach in experiments. For future work the preview control based reference generation method will be used for gait transitions.

TABLE I. Simulation Model Parameters

\begin{tabular}{ccc}
\hline \hline Joint & Dimensions $(\mathrm{LxWxH})[\mathrm{m}]$ & Mass $[\mathrm{kg}]$ \\
\hline Trunk & $1.2 \times 0.6 \times 0.15$ & 50 \\
Thigh & $0.28 \times 0.05 \times 0.1$ & 4.8 \\
Shank & $0.27 \times 0.05 \times 0.1$ & 3.85 \\
Ankle & $0.22 \times 0.05 \times 0.5$ & 3.85
\end{tabular}


LOCOMOTION PARAMETERS

\begin{tabular}{ccc}
\hline \hline Symbo & Definition & Value \\
\hline$T_{s}$ & Step duration & $0.5 \mathrm{~s}$ \\
$A$ & $y$-coordinate of foot location & $0.20 \mathrm{~m}$ \\
$2 B$ & Step length & $0.20 \mathrm{~m}$ \\
$h_{s}$ & Step height & $0.05 \mathrm{~m}$
\end{tabular}
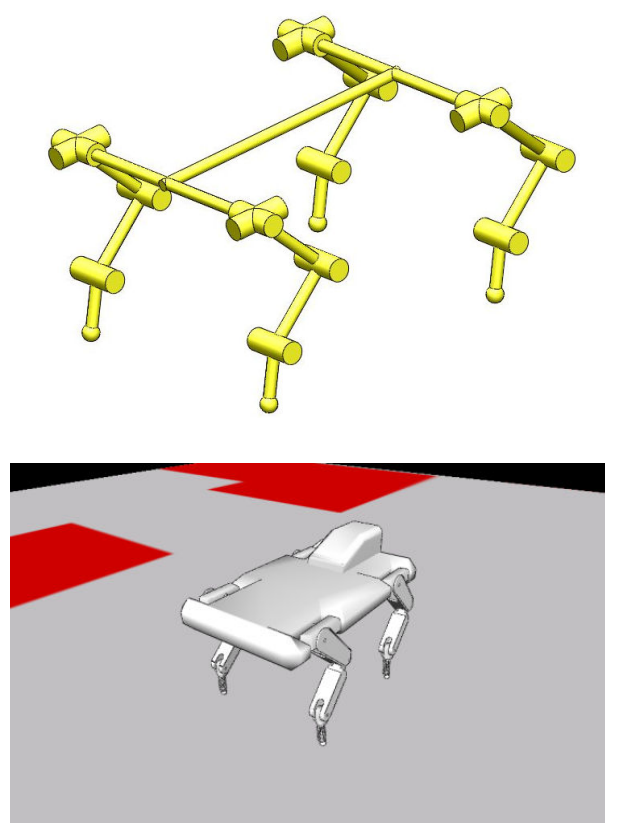

Figure 7. Kinematic arrangement and animation environment

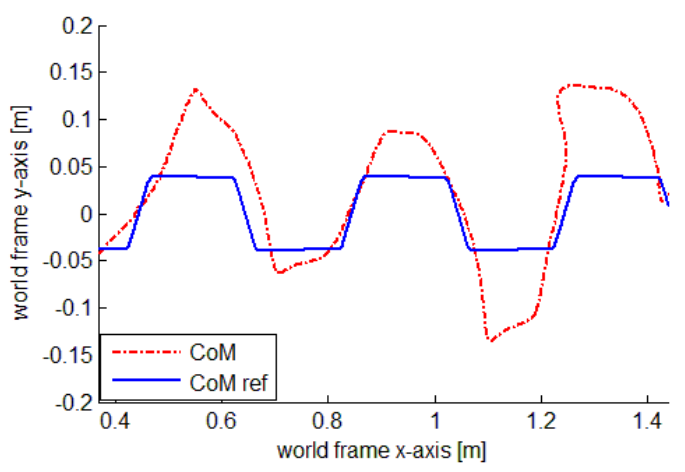

Figure 8. CoM reference trajectory and actual CoM trajectory wrt. world coordinate frames.
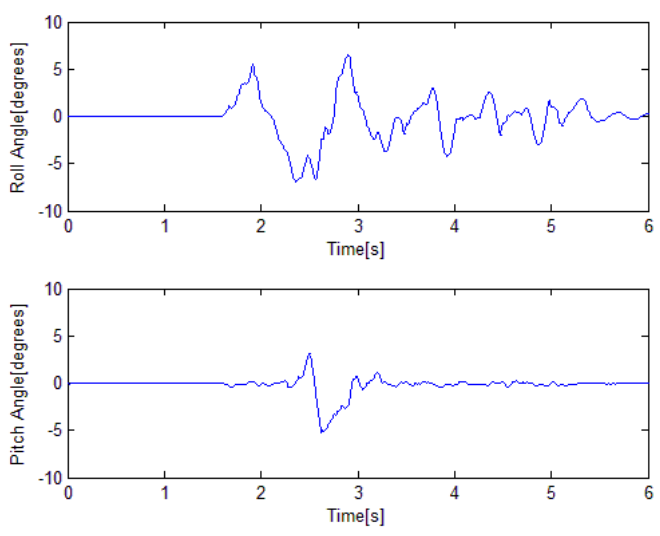

Figure 9. Roll and pitch angles for pace gait consist of 7 steps. This figure corresponds to the trajectory references in Figure 5.

\section{REFERENCES}

[1] S. Hirose, Y. Fukuda, K. Yoneda, A. Nagakubo, H. Tsukagoshi and K. Arikawa, et al. "Quadruped Walking Robots at Tokyo Institude of Technology," IEEE Robotics and Automation Magazine, pp:104-114, 2009.

[2] S. Hirose, K. Yoneda, R. Furuya, and T. Takagi, "Dynamic and static fusion gait of a quadruped walking vehicle," In Proc. of IEEE/RSJ Int. Conf. on Intelligent Robots and Systems '89, pp: 199-204, 1989.

[3] K. Yoneda, H. Iiyama ve S Hirose, "Intermittent Trot Gait of a Quadruped Walking Machine Dynamic Stability Control of an Omnidirectiona Walk," In Proc. Int. Conf. on Robotics and Automation, pp: 3002-3007, 1996.

[4] K. Yoneda and S. Hirose, "Dynamic and static fusion gait of a quadruped walking vehicle on a winding path," Advanced Robotics, pp: 125-136, 1994.

[5] R. Kurazume, K. Yoneda and S. Hirose "Feedforward and feedback dynamic trot gait control for quadruped walking vehicle," Autonomous Robots, Vol: 12, No: 2, pp: 157-172, 2002.

[6] H. Takeuchi, "Real Time Optimization for Robot Control using Receding Horizon Control with Equal Constraint," Journal of Robotics Systems, Vol: 20, pp: 3-13, 2003.

[7] H. Osumi, S. Kamiya, H. Kato, K. Umeda, R. Ueda and T. Arai, "Time optimal control for quadruped walking robots," IEEE International Conference on Robotics and Automation, pp:1102-1108, 2006.

[8] H. Kimura, Y. Fukuoka, and A. Cohen, "Biologically inspired adaptive walking of a quadruped robot," Phil. Trans. R. Soc., 2007, Vol:365, pp: $153-170$

[9] P. Buono and M. Golubitsky, "Models of central pattern generators for quadruped locomotion,” J. Math. Biol., Vol: 42, pp:291-326, 2001

[10] S. Hornby, M. Fujita, S. Takamura, T. Yamamoto and O. Hanagata, "Autonomous Evolution of Gaits with the Sony Quadruped Robot," Proc. of the 1999 Genetic and Evolutionary Computation Conference

[11] M. Hardti and O von Stryk, "Towards Optimal Hybrid Control Solutions for Gait Patterns of a Quadruped," Proc. Int. Conf. on Climbing and Walking Robots, 2000

[12] R. Kurazume, A. Byong-won, K. Ohta, and T. Hasegawa, "Esperimental Study on Energy Efficiency for Quadruped Walking Vehicles," in Proc. of the 2003 IEEE/RSJ, Las Vegas, 2003

[13] K. Erbatur and O. Kurt, "Natural ZMP trajectories for Biped Robot Reference Generation," IEEE Transactions on Industrial Electronics, Vol: 56, No: 3, 2009.

[14] K. Sorao, T. Murakami and K. Ohnishi, "A Unified Approach to ZMP and Gravity Center Control in Biped Dynamic Stable Walking," Proceedings of IEEE ASME Int. Conf. Adv Intelligent Mechatronics, CD-Rom, 1987. 
[15] J.H.Park, and Y.K. Rhee, "ZMP Trajectory Generation for Reduced Trunk Motions of Biped Robots," Proc. IEEE IRSJ Int. Conf Intelligent Robots and Systems, IROS '98 pp: 90-95, 1998.

[16] M. Vukobratovic, B. Borovac, D. Surla, and D. Stokic, Biped Locomotion: Dynamics, Stability and Application. Berlin, Germany: Springer-Verlag, 1990

[17] S. Kajita, F. Kahehiro, K. Kaneko, K. Fujiwara, K. Harada, K. Yokoi and H. Hirukawa, " Biped Walking Pattern Generation using Preview Control of the Zero-Moment-Point," Proceedings of IEEE International Conference on Robotics and Automation, Vol:2, pp:1620-1626, 2003

[18] E. Taskiran, M. Yilmaz, O. Koca, U. Seven and K.Erbatur, "Trajectory Generation with Natural ZMP References for the Biped Walking Robot SURALP," IEEE International Conference on Robotics and Aut. pp: 4237-4242, 2010.

[19] J. Y. Kim, I. W. Park, J. H. Oh, "Walking Control Algorithm of Biped Humanoid Robot on Uneven and Inclined Floor," J Intell Robot Syst (2007) 48:457-484, January 2007

[20] K. Erbatur, U. Seven, E. Taskiran, Ö. Koca, M. Yilmaz, G. Kızıltas, M. Ünel, A. Sabanovic, A. Onat, "SURALP: A New Full-Body Humanoid Robot Platform," IEEE/RSJ International Conference on Intelligent Robots and Systems, IROS 2009, St. Louis, MO, USA, Ekim 2009.

[21] H. Takeuchi, "Development of MEL HORSE," IEEE International Conference on Robotics and Automation pp: 3165-3170, 2001.

[22] W. Pengfei, H. Bo and S. Lining, "Walking research on multi-motion mode quadruped bionic robot based on moving ZMP," IEEE International Conference on Mechatronics \& Automation, Vol:4, pp:1935-1940, 2005.

[23] M. Kalakrishnan, J. Buchli, P. Pastor, M. Mistry and S. Schaal, "Fast, robust quadruped locomotion over challenging terrain," IEEE International Conference on Robotics and Automation, pp:2665-2670, 2010.

[24] C. Theeravithayangkura, T. Takubo, K. Ohara, Y. Mae and T. Arai, "Dynamic Rolling-walk Motion by Limb Mechanism Robot ASTERISK," IEEE International Conference on Mechatronics and Automation, pp:2659-2664, 2009.

[25] K. Byl, A. Shkolnik, S. Prentice, N. Roy and R. Tedrak, "Reliable Dynamic Motions for a Stiff Quadruped," Springer Tracks in Advanced Robotics, Vol:54, pp:319-328, 2009

[26] Y. Choi, B. J. You, and S. R. Oh, "On the stability of indirect ZMP controller for biped robot systems," Proc. of Int. Conf. on Intelligent Robots and Systems, pp: 1966-1971, vol.2, Sendal, Japan, June 2004.

[27] B. Verrelst, O. Stasse, K. Yokoi, B. Vanderborght, "Dynamically Stepping Over Obstacles by the Humanoid Robot HRP-2," Proc. IEEE/RAS International Conference on Humanoid Robots, CenovaItalya, Aralık 2006

[28] K. C. Fidan, T. Akbaș, Ș. E. Eskimez, S. Özel, Ö. K. Adak, Ö. Drama, M. Konukoğlu, G. Yılmaz, K. Erbatur, "Dört Bacaklı Robotlar için Önizlemeli Kontrol ile Sıfır Moment Noktası Tabanlı Yürüme Yörüngesi Sentezi-ZMP Based Locomotion Trajectory Synthesis for Quadruped Robots with Preview Control," Turkish Automatic Control Conference, September 2011, Izmir, Turkey, (In Turkish)

[29] M. Tomizuka, Rosenthal, D.E, "On the Optimal Digital State Vector Feedback Controller with Integral and Preview Actions," Trans. of the ASME, J. of Dyn. Sys. Meas. Contr., 101, pp.172-178, 1979.

[30] T. Katayama, Ohki, T., Inoue, T. and Kato, T., "Design of an Optimal Controller for a Discrete Time System Subject to Previewable Demand," Int. J. Control, Vol.41, No.3, pp.677-699, 1985.

[31] K. Erbatur, A. Kawamura, "A New Penalty Based Contact Modeling and Dynamics Simulation Method as Applied to Biped Walking Robots," CD Proc. FIRA World Congress 2003,Vienna, Austria 